Article

\title{
Computerized Cytological Features for Papillary Thyroid Cancer Diagnosis-Preliminary Report
}

\author{
Shyang-Rong Shih ${ }^{1,2,3}$, I-Shiow Jan ${ }^{4}$, Kuen-Yuan Chen ${ }^{5}$, Wan-Yu Chuang ${ }^{6}$, \\ Chih-Yuan Wang ${ }^{1,2}{ }^{-}$, Yung-Lien Hsiao ${ }^{2}$, Tien-Chun Chang ${ }^{1,3,7, *}$ and Argon Chen ${ }^{8, *}$ \\ 1 National Taiwan University College of Medicine, Taipei 10051, Taiwan; srshih@ntu.edu.tw (S.-R.S.); \\ cyw1965@gmail.com (C.-Y.W.) \\ 2 Department of Internal Medicine, National Taiwan University Hospital, Taipei 10002, Taiwan; \\ hsaio17@yahoo.com.tw \\ 3 Center of Anti-Aging and Health Consultation, National Taiwan University Hospital, Taipei 10002, Taiwan \\ 4 Department of Laboratory Medicine, National Taiwan University Hospital, Taipei 10002, Taiwan; \\ isjan15719@ntu.edu.tw \\ 5 Department of Surgery, National Taiwan University Hospital, Taipei 10002, Taiwan; dtsurg51@gmail.com \\ 6 AmCad BioMed Corporation, Taipei 10547, Taiwan; rachel.chuang@amcad.com.tw \\ 7 Far Eastern Polyclinic, Taipei 10043, Taiwan \\ 8 Institute of Industrial Engineering, National Taiwan University, Taipei 10617, Taiwan \\ * Correspondence: tienchunchang@ntu.edu.tw (T.-C.C.); achen@ntu.edu.tw (A.C.); \\ Tel.: +886-2-3366-9504 (A.C.); Fax: +886-2-2362-5856 (A.C.)
}

Received: 15 September 2019; Accepted: 21 October 2019; Published: 25 October 2019

\begin{abstract}
Fine needle aspiration cytology (FNAC) is the final diagnosis of thyroid nodules before surgery. It is important to further improve the indeterminate FNAC diagnosis results using computerized cytological features. This retrospective cross-sectional study included 240 cases, of whom 110 had histologic diagnosis of papillary thyroid cancers (PTC), 100 had nodular/adenomatous goiters/hyperplasia (benign goiters), 10 had follicular/Hurthle cell carcinomas, and 20 had follicular adenomas. Morphological and chromatic features of FNAC were quantified and analyzed. The result showed that six quantified cytological features were found significantly different between patients with a histologic diagnosis of PTC and patients with histologic diagnosis of benign goiters in multivariate analysis. These cytological features were used to estimate the malignancy risk in nodules with indeterminate FNAC results. The Area Under the Receiver Operating Characteristics (AUROC) of the diagnostic accuracy with a benign or malignant nature was $81.3 \%$ $(p<0.001), 78.7 \%(p=0.014)$, and $56.8 \%(p=0.52)$ for nodules with FNAC results of atypia, which is suspicious for malignancy and follicular neoplasm, respectively. In conclusion, quantification of cytological features could be used to develop a computer-aided tool for diagnosing PTC in thyroid nodules with indeterminate FNAC results.
\end{abstract}

Keywords: thyroid cytology; diagnosis; computer analysis; morphometry

\section{Introduction}

Thyroid nodules are common. They can be detected in 19\%-67\% of the normal population using high-resolution sonography, and 5\%-15\% are malignant [1]. Fine needle aspiration cytology (FNAC) has an essential role in evaluating thyroid nodules before surgery. In samples satisfactory for interpretation, $2 \%-5 \%$ are reported as definitively malignant and $55 \%-74 \%$ as definitively benign [2-5]. The remaining samples have indeterminate cytology, including atypia of undetermined significance or follicular lesion of undetermined significance (AUS/FLUS) (2\%-18\%), follicular neoplasm or suspicious for a follicular neoplasm (FN/SFN) (2\%-25\%), and suspicious for malignancy (SUSP) (1\%-6\%) [6]. Therefore, 
researchers are seeking methods to further improve preoperative diagnosis or prognostic prediction of thyroid nodules, including genetic evaluation and computerized cytological morphometry [6-8]. Commercial molecular tests for thyroid FNAC include ThyroSeq, Afirma, RosettaGX Reveal, ThyGenX, and ThyraMIR [9]. Their sensitivity for the diagnosis of thyroid cancer ranges from $74 \%$ to $91 \%$, with specificity from $49 \%$ to $93 \%$, negative predictive value (NPV) from $92 \%$ to $97 \%$, and positive predictive value (PPV) from $37 \%$ to $83 \%$ in tumors with cytological categories of AUS/FLUS and FN. The molecular approach is an ideal "rule-in" test if the PPV for histopathologically-proven malignancy is high. On the other hand, the molecular approach is an ideal "rule out" test if the NPV is high. Most molecular tests listed above have high NPV and lower PPV [6]. The reproducibility of some genetic tests measured in different populations has been questioned [10-12]. Moreover, the molecular tests are expensive and not widely available. Computerized cytomorphometry enables objective quantification of selected morphologic and chromatic parameters in individual cells [13]. It serves as a sequential reader to the conventional cytological evaluation. We aimed to analyze the cytological features of benign and malignant thyroid nodules using computerized cytomorphometry to aid in the differential diagnosis of FNAC. Papillary thyroid cancer (PTC) is the most common thyroid malignancy and constitutes up to $90 \%$ of differentiated follicular cell-derived thyroid cancers (FCTC) worldwide [14]. In this preliminary study, we focused on the cytological features of PTC and the other type of FCTC, namely, follicular thyroid cancer (FTC), in comparison with those benign follicular cell-derived lesions.

\section{Results}

Table 1 shows demographic characteristics, according to categories of cytological diagnoses. Malignant tumors accounted for $13.33 \%, 57.5 \%, 31.25 \%, 94.34 \%$, and $100 \%$, respectively, of the benign, AUS, FN/SFN, SUSP, and malignant categories on cytological diagnosis. Patients' age and sex were not significantly different between the pathologically diagnosed benign and malignant tumors in each of the cytological categories. In patients with pathologic diagnosis of PTC, five cases had follicular variants of PTC (FVPTC). Three of them had non-encapsulated subtype of FVPTC (NFVPTC) and two had mixed classical type of PTC (cPTC) and NFVPTC. The other 105 PTCs were cPTC.

\subsection{Diagnosis of Papillary Thyroid Cancer (PTC) and Benign Follicular Lesions Other than Adenoma}

In pathologic diagnosis of PTC and benign nodular/adenomatous goiter/hyperplasia, eight cytological features in FNAC specimens were significantly different between pathologically benign and malignant nodules, including mean nuclear size (MNS), standard deviation of nuclear size (SDNS), mean of nuclear elongation (MNElon), coefficient of variation of nuclear elongation, nuclear-cytoplasmic saturation ratio (NCSR), nuclear-cytoplasmic ratio (NCR), nuclear polarity (NP), and inclusion Index (II) (Table 2).

These eight features were then analyzed using stepwise logistic regression with the $p$-value of entering variables set at 0.05 and the $p$-value of removing variables set at 0.1 . Six features, i.e., MNS, MNElon, NCSR, NCR, NP, and II, remained significant and were retained in the model. A logistic regression model was then built using these six cytological features as independent variates and pathologic diagnosis as the dependent variate (benign: 0, malignant: 1) (Table 3).

With the logistic regression model, the probability of malignancy could then be estimated using the following. Probability $=\frac{1}{1+e^{\log i t(p)}}$ where $\operatorname{logit}(p)=-9.71158+0.06557 \mathrm{MNS}+8.94005 \mathrm{MNElon}-$ $1.27953 \mathrm{NCSR}+1.895 \mathrm{NCR}+0.038098 \mathrm{NP}+32.1987 \mathrm{II}$. 
Table 1. Basic characteristics of the study subjects.

\begin{tabular}{|c|c|c|c|c|c|c|c|c|c|c|c|c|c|}
\hline \multirow{2}{*}{$\begin{array}{l}\text { Cytological Diagnosis } \\
\text { (Bethesda Category) }\end{array}$} & \multicolumn{3}{|c|}{ Benign } & \multicolumn{3}{|c|}{$\begin{array}{l}\text { Atypia of Undetermined Significance or } \\
\text { Follicular Lesion of Undetermined } \\
\text { Significance (AUS) }\end{array}$} & \multicolumn{3}{|c|}{$\begin{array}{l}\text { Follicular Neoplasm or Suspicious for a } \\
\text { Follicular Neoplasm (FNSFN) }\end{array}$} & \multicolumn{3}{|c|}{ Suspicious for Malignancy (SUSP) } & \multirow{2}{*}{$\begin{array}{c}\text { Malignant } \\
\text { Malignant (\%) }\end{array}$} \\
\hline & Benign & Malignant (\%) & $p$ & Benign & Malignant (\%) & $p$ & Benign & Malignant (\%) & $p$ & Benign & Malignant (\%) & $p$ & \\
\hline Number & 78 & $12(13.33 \%)$ & & 17 & $23(57.5 \%)$ & & 22 & $10(31.25 \%)$ & & 3 & $50(94.34 \%)$ & & $25(100 \%)$ \\
\hline Age (SD) & $52(12)$ & $50(19)$ & 0.525 & $46(14)$ & $44(11)$ & 0.564 & $55(12)$ & $54(20)$ & 0.817 & $50(29)$ & $52(11)$ & 0.868 & $51(15)$ \\
\hline Gender (Female: Male) & 69:9 & $10: 2$ & 0.629 & 13:4 & $16: 7$ & 0.645 & $18: 4$ & $6: 4$ & 0.215 & 3:0 & $38: 12$ & 0.355 & $22: 3$ \\
\hline
\end{tabular}

$p$-values were calculated by independent samples $t$-test for the age and by Mann-Whitney test for the gender. 
Table 2. Cytomorphometry of fine needle aspiration specimens in different pathologic diagnosis.

\begin{tabular}{|c|c|c|c|c|c|}
\hline \multirow[b]{2}{*}{ Pathologic Diagnosis } & \multicolumn{2}{|c|}{ Papillary Thyroid Cancer and Benign Follicular Lesions other than Follicular Adenoma } & \multicolumn{2}{|c|}{ Follicular Neoplasm } & \multirow[b]{2}{*}{$p^{*}$} \\
\hline & $\begin{array}{c}\text { Nodular Hyperplasia/Goiter, Adenomatous } \\
\text { Hyperplasia/Goiter (Group 1) }\end{array}$ & Papillary Thyroid Cancer (Group 2) & Follicular Adenoma (Group 3) & $\begin{array}{c}\text { Follicular Carcinoma Hurthle } \\
\text { Cell Carcinoma (Group 4) }\end{array}$ & \\
\hline Number & 100 & 110 & 20 & 10 & \\
\hline Age (year) & $53(13)$ & $50(13)$ & $48(14)$ & $48(19)$ & 0.358 \\
\hline Gender (Female: Male) & $87: 13$ & $85: 25$ & $16: 4$ & 7:3 & 0.247 \\
\hline Mean nuclear size $\left(\mu \mathrm{m}^{2}\right)$ & $56.03(43.46-74.93)$ & $75.07(65.67-87.15)$ & $81.42(60.69-95.13)$ & $77.60(58.38-98.14)$ & $<0.001^{+, \ddagger, \S}$ \\
\hline Standard deviation of nuclear size $\left(\mu \mathrm{m}^{2}\right)$ & $8.02(5.06-11.23)$ & $10.57(8.16-14.36)$ & $12.92(5.90-16.02)$ & $12.71(8.95-20.16)$ & $0.007^{\dagger}$ \\
\hline Coefficient of variation of nuclear size & $0.150(0.095-0.214)$ & $0.144(0.114-0.192)$ & $0.161(0.077-0.292)$ & $0.163(0.137-0.180)$ & 0.927 \\
\hline Mean nuclear circularity & $0.839(0.819-0.851)$ & $0.832(0.817-0.840)$ & $0.846(0.831-0.861)$ & $0.839(0.811-0.852)$ & 0.062 \\
\hline Standard deviation of nuclear circularity & $0.019(0.014-0.029)$ & $0.021(0.015-0.031)$ & $0.014(0.011-0.023)$ & $0.020(0.015-0.024)$ & 0.632 \\
\hline Coefficient of variation of nuclear circularity & $0.023(0.016-0.036)$ & $0.025(0.018-0.038)$ & $0.016(0.013-0.027)$ & $0.024(0.018-0.030)$ & 0.672 \\
\hline Mean nuclear elongation & $0.505(0.457-0.559)$ & $0.546(0.503-0.569)$ & $0.478(0.453-0.541)$ & $0.506(0.486-0.572)$ & $<0.001^{+, \|}$ \\
\hline Standard deviation of nuclear elongation & $0.067(0.049-0.080)$ & $0.062(0.051-0.077)$ & $0.066(0.046-0.098)$ & $0.069(0.054-0.078)$ & 0.11 \\
\hline Coefficient of variation of nuclear elongation & $0.134(0.099-0.165)$ & $0.115(0.092-0.141)$ & $0.133(0.098-0.196)$ & $0.120(0.108-0.154)$ & $0.012^{+}$ \\
\hline Mean nuclear ellipticity & $0.872(0.852-0.881)$ & $0.871(0.860-0.879)$ & $0.880(0.864-0.892)$ & $0.876(0.861-0.889)$ & $0.017 \ddagger$ \\
\hline Standard deviation of nuclear ellipticity & $0.014(0.010-0.021)$ & $0.015(0.011-0.020)$ & $0.014(0.008-0.019)$ & $0.014(0.012-0.023)$ & 0.528 \\
\hline Coefficient of variation of nuclear ellipticity & $0.015(0.011-0.024)$ & $0.017(0.012-0.024)$ & $0.015(0.009-0.022)$ & $0.016(0.014-0.027)$ & 0.556 \\
\hline Nuclear-to-cytoplasmic hue ratio & $1.024(1.011-1.043)$ & $1.036(1.016-1.053)$ & $1.015(0.998-1.026)$ & $1.027(1.012-1.053)$ & $0.001 \|$ \\
\hline $\begin{array}{l}\text { Standard deviation of } \\
\text { Nuclear-to-cytoplasmic hue ratio }\end{array}$ & $0.985(0.864-1.087)$ & $0.983(0.885-1.116)$ & $0.901(0.822-0.984)$ & $0.902(0.830-0.990)$ & 0.082 \\
\hline Nuclear-to-cytoplasmic saturation ratio & $1.944(1.652-2.422)$ & $1.693(1.570-1.979)$ & $2.232(1.914-2.347)$ & $2.322(1.731-2.566)$ & $<0.001+, \|$ \\
\hline $\begin{array}{l}\text { Standard deviation of } \\
\text { Nuclear-to-cytoplasmic saturation ratio }\end{array}$ & $0.911(0.797-1.109)$ & $0.908(0.774-1.013)$ & $0.937(0.813-1.152)$ & $0.920(0.848-0.991)$ & 0.105 \\
\hline Nuclear-to-cytoplasmic value ratio & $0.833(0.791-0.872)$ & $0.835(0.805-0.858)$ & $0.798(0.756-0.863)$ & $0.864(0.789-0.869)$ & 0.232 \\
\hline $\begin{array}{l}\text { Standard deviation of } \\
\text { Nuclear-to-cytoplasmic value ratio }\end{array}$ & $1.086(0.935-1.194)$ & $1.099(0.986-1.286)$ & $0.987(0.933-1.158)$ & $1.133(0.945-1.227)$ & 0.506 \\
\hline Nuclear-to-cytoplasmic ratio & $0.998(0.740-1.373)$ & $1.230(1.020-1.556)$ & $1.056(0.755-1.806)$ & $0.941(0.806-1.114)$ & $0.025^{\dagger}$ \\
\hline Nuclear polarity & $14.02(5.04-23.49)$ & $22.48(15.27-32.69)$ & $13.09(4.19-18.26)$ & $12.75(7.56-19.01)$ & $<0.001^{+, \|}$ \\
\hline Inclusion index & $0(0.000-0.004)$ & $0.006(0.002-0.012)$ & $0.004(0.000-0.007)$ & $0.007(0.005-0.018)$ & $0.011^{\dagger}$ \\
\hline Overlapping index & $0.436(0.340-0.577)$ & $0.500(0.432-0.589)$ & $0.473(0.290-0.581)$ & $0.452(0.374-0.596)$ & 0.221 \\
\hline
\end{tabular}

Data are presented as mean $\pm \mathrm{SD}$ if the continuous variable is normally distributed, and as a median (interquartile range) if not normally distributed. * $\mathrm{P}$ was obtained using the ANOVA

test to compare the difference between the four groups. ${ }^{\dagger}$ The results are significantly different between group 1 and group $2, \neq$ significantly different between group 1 and group 3 ,

$\S$ significantly different between group 1 and group 4 , and " significantly different between group 2 and group 3 when using the Scheffé test for all pairwise comparisons. 
Table 3. The relationship between pathologic diagnosis and cytological characteristics in pathologic diagnosis of papillary thyroid cancer and benign follicular lesions other than follicular adenoma. Multivariate logistic regression analysis was performed using pathological diagnosis as the dependent variate (malignant: 1, benign: 0$)$ and six cytological characteristics as independent variates $(n=210)$.

\begin{tabular}{ccc}
\hline Variable & Coefficient & $p$ \\
\hline Mean nuclear size $\left(\mu \mathrm{m}^{2}\right)$ & 0.06557 & $<0.0001$ \\
Mean nuclear elongation & 8.94005 & 0.0239 \\
Nuclear-to-cytoplasmic saturation ratio & -1.27953 & 0.0008 \\
Nuclear-to-cytoplasmic ratio & 1.89500 & $<0.0001$ \\
Nuclear polarity & 0.038098 & 0.0259 \\
Inclusion index & 32.19870 & 0.0473 \\
Constant & -9.71158 & 0.0001 \\
\hline
\end{tabular}

With the estimated probability of malignancy calculated for each case, the receiver operating characteristic (ROC) curve analysis was performed. In pathologic diagnosis of papillary thyroid cancer and benign follicular lesions other than follicular adenoma, the value for the area under the ROC curve (AUROC) was 87.7\% ( $p<0.001)$ (Figure 1A). The threshold probability for making diagnosis was then calculated to be 0.535 based on the optimum cut-off point on the ROC curve closest to $(0,1)$. The corresponding diagnosis sensitivity, specificity, PPV, and NPV were 84.5\%, 81.0\%, 82.3\%, and $82.5 \%$, respectively. Figure 2 shows the differentiation performance of the logistic model using box plots for different cytological categories.
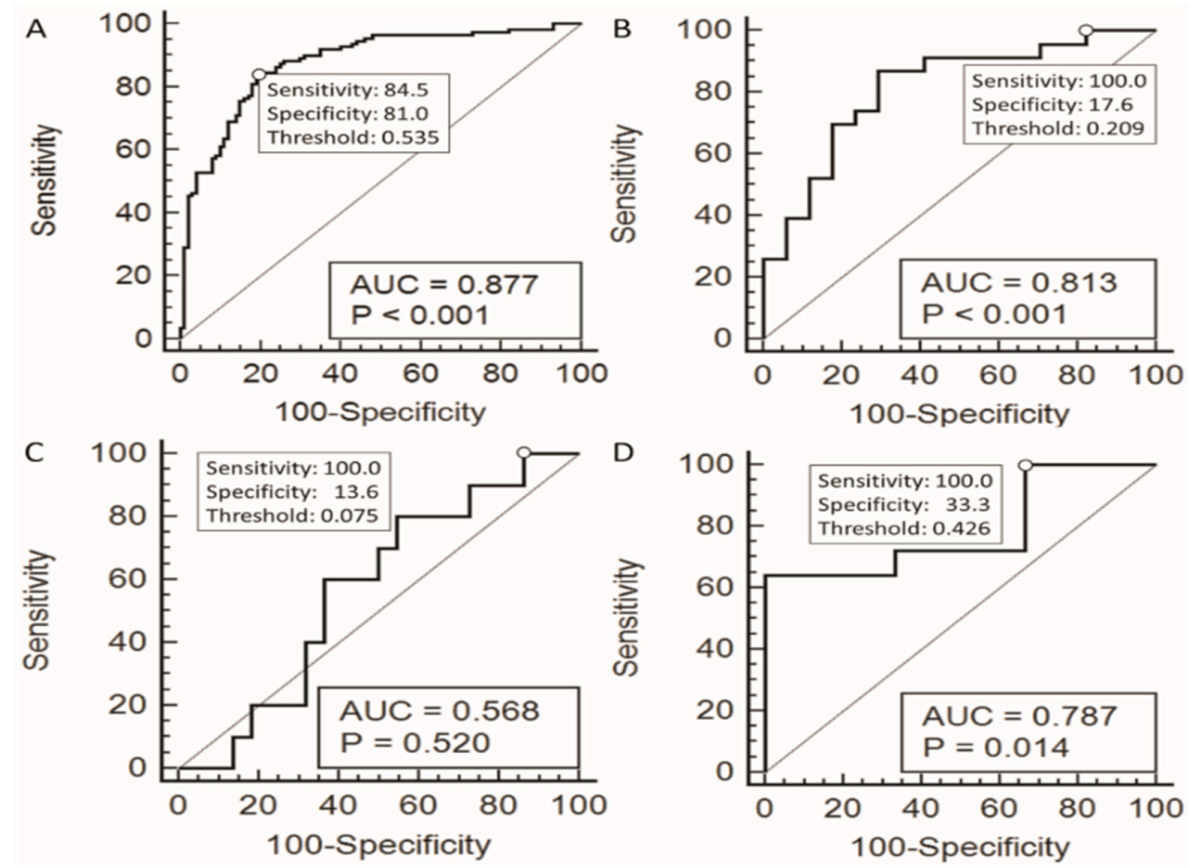

Figure 1. Receiver operating characteristic curves and their area under the curve (AUC), where positive and negative outcomes were defined as malignant and benign, respectively. (A) In pathologic diagnosis of papillary thyroid cancer and benign follicular lesions other than follicular adenoma $(n=210)$. (B) In Bethesda cytological category of atypia of undetermined significance or follicular lesion of undetermined significance $(n=40)$. (C) In Bethesda cytological category of follicular neoplasm or suspicious for a follicular neoplasm $(n=32)$. (D) In Bethesda cytological category of suspicious for malignancy $(n=53)$. In panel $\mathrm{A}$, the threshold value was set at the cut-off point that was closest to $(0,1)$. In panel B, C, and D, the threshold value was set at the sensitivity that was $100 \%$ aligned with specificity that is as high as possible. 


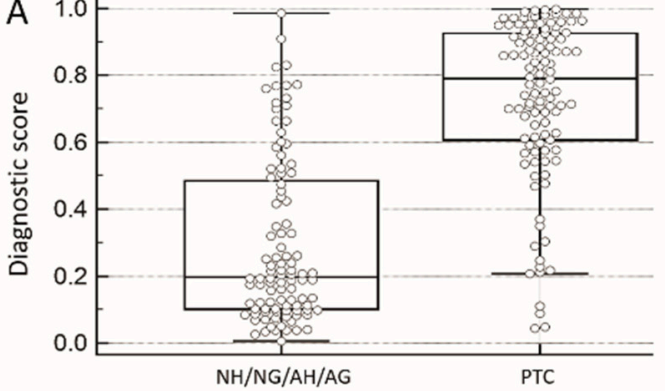

Pathologic diagnosis

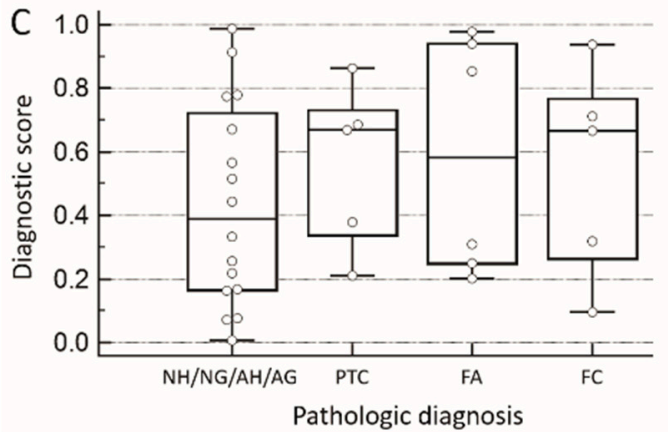

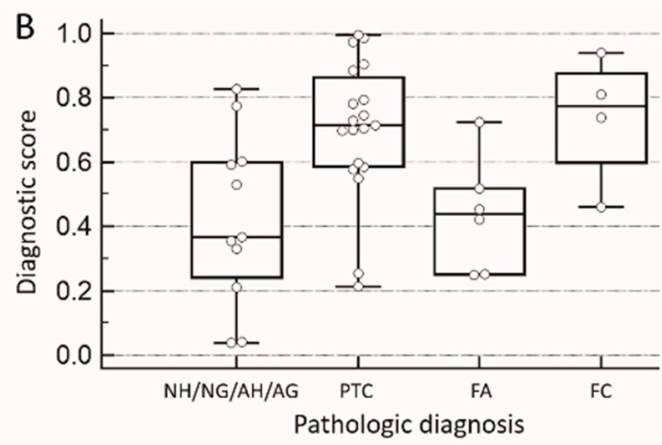

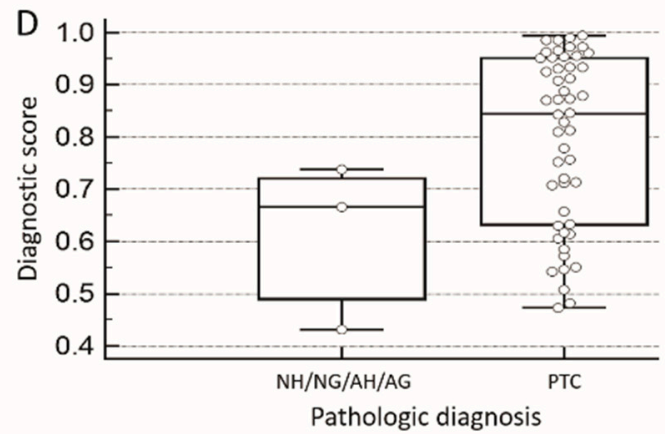

Figure 2. Box plots of the relationship between a diagnostic score and pathologic diagnosis. (A) In pathologic diagnosis of papillary thyroid cancer and benign follicular lesions other than follicular adenoma $(n=210)$. (B) In Bethesda cytological category of atypia of undetermined significance or follicular lesion of undetermined significance $(n=40)$. (C) In Bethesda cytological category of follicular neoplasm or suspicious for a follicular neoplasm $(n=32)$. (D) In Bethesda cytological category of suspicions for malignancy $(n=53)$. NH: Nodular hyperplasia. NG: Nodular goiter. AH: Adenomatous hyperplasia. AG: Adenomatous goiter. PTC: Papillary thyroid cancer. FA: Follicular adenoma. FC: Follicular carcinoma.

\subsection{Performance of the Logistic Model in Cytologically Indeterminate Categories}

The ROC curve analyses were performed for cases in the indeterminate AUS, FN/SFN, and SUSP cytological categories and their AUROC values were $81.3 \%(p<0.001), 56.8 \%(p=0.52)$, and $78.7 \%$ $(p=0.014)$, respectively (Table 4$)$. The optimum threshold probabilities based on the cut-off point closest to $(0,1)$ for the AUS, FN/SFN, and SUSP categories were calculated to be $0.523,0.304$, and 0.733 , with (sensitivity percentage, specificity percentage) equal to $(87.0,70.6),(80.0,45.5)$, and $(64.0,100.0)$, respectively (Table 4, model 2). The logistic model was more sensitive for the AUS category and was very specific for the SUSP category, while the model was not as effective for FN/SFN cases.

Table 4. Prediction of pathologic diagnosis of thyroid cancer using a diagnostic score.

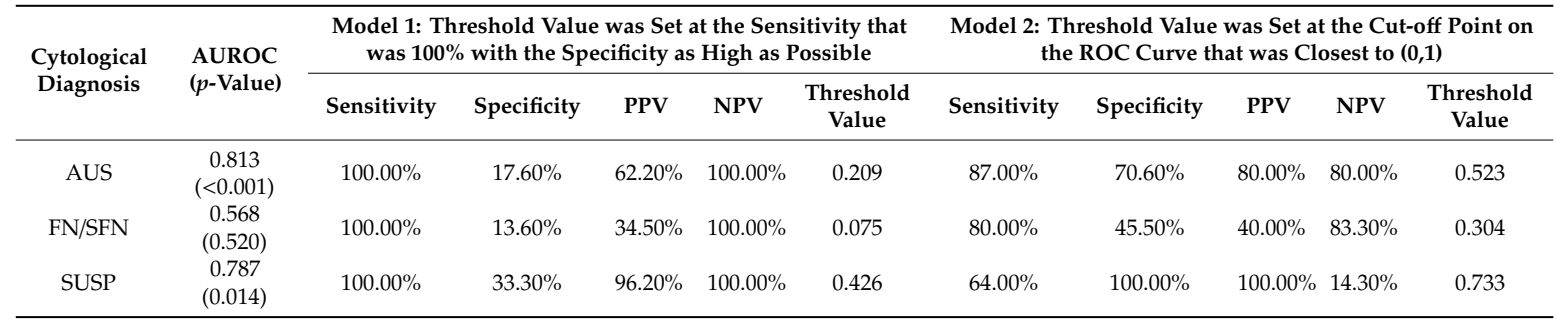

PPV: positive predictive value. NPV: negative predictive value. AUS: atypia of undetermined significance or follicular lesion of undetermined significance. FN/SFN: follicular neoplasm or suspicious for a follicular neoplasm. SUSP: suspicious for malignancy.

To assist the cytologist in further differentiating the benign cases from the indeterminate cases, one may choose the threshold probabilities to ensure a sensitivity of $100 \%$, i.e., no malignant cases 
missed, with the specificity as high as possible. Table 4 shows the diagnosis results under various threshold probabilities set at different cut-off points of the ROC curves. With the cut-off points ensuring $100 \%$ sensitivity, it can be seen that the threshold probability values were $0.209,0.075$, and 0.426 for the AUS, FN/SFN, and SUSP categories and the specificities can reach $17.6 \%, 13.6 \%$, and $33.3 \%$, respectively (Figure 1 panel B, C, and D, Table 4 and model 1). With the assistance of the logistic model constructed by the computerized cytological features, 3 out of 17 benign AUS cases, 3 out of 22 benign FN/SFN cases, and 1 out of 3 benign SUSP cases might have avoided aggressive treatment, such as surgical thyroidectomy.

\section{Discussion}

This study proposed several new parameters for computerized morphometry of thyroid FNAC specimens. We can quantify not only morphological but also chromatic features. Our study shows that computerized quantification of cytological characteristics can help to differentiate $17.6 \%-33.3 \%$ of cases as benign rather than malignant, with $100 \%$ sensitivity, for the Bethesda categories of AUS and SUSP. Even for the FN/SFN category, $13.6 \%$ of cases can be further differentiated as benign with $100 \%$ sensitivity, using a model based on PTC cases and benign nodular/adenomatous goiter/hyperplasia. This is because the model is designed to differentiate not only PTC nodules but also benign nodular adenomatous goiter and hyperplasia. Among the three of 22 benign cases of FN/SFN differentiated with the model, one was an adenomatous goiter and two were hyperplastic nodules. The cytological features used in this model for differential diagnosis included mean nuclear size (MNS), mean nuclear elongation (MNElon), nuclear-to-cytoplasmic saturation ratio (NCSR), nuclear-to-cytoplasmic ratio (NCR), nuclear polarity (NP), and inclusion index (II).

Much effort has focused on genetic analysis to aid in diagnosis of indeterminate cytology reports for thyroid nodules. BRAF, NRAS, HRAS, and KRAS point mutations, and RET/PTC1, RET/PTC3, and PAX8/PPARC rearrangements were reported to be helpful [6]. Several commercial thyroid FNAC molecular tests are available, including ThyroSeq, Afirma, RosettaGX Reveal, ThyGenX, and ThyraMIR [9]. However, genetic examination is expensive and not widely available. We previously demonstrated that computerized morphometry of FNAC samples helps predict recurrence of PTC [7]. The nucleus-cell ratio and variation of nuclear area showed significant and positive correlations with recurrence ( $p=0.002$ and 0.044 , respectively). The present study quantified cytological features and identified differences between benign and malignant thyroid nodules. Larger nuclear size and higher nuclear-to cytoplasmic ratio are related to malignancy. This may be related to cellular duplication activity [8], and was reportedly associated with prognosis for several cancers, including malignant melanoma, rhabdomyosarcoma, and renal cell carcinoma [15-17]. Our study also showed that NCSR is related to diagnosis. PTC cells show less difference in saturation between nuclei and cytoplasm than benign follicular cells. There has been no other description of the association between this chromatic feature and malignancy in the literature. PTC cells had greater mean nuclear elongation than benign follicular cells in our study. This also had not been mentioned in the research. PTC cells are usually found in a papillaroid arrangement. In the cross section of each papilla, the elongated cells are arranged in a spiral shape, which results in a different direction for each cell and greater nuclear polarity in PTC. Inclusion bodies are characteristic features of PTC, and, therefore, the index of inclusion is higher in PTC.

This computerized diagnostic score was developed for the purpose of assisting cytopathologists and clinicians in challenging situations of indeterminate thyroid cytology. According to the American Thyroid Association guideline, in the situation of indeterminate thyroid cytologic results, clinicians should discuss with the patients to decide further management, including repeated FNAC, genetic study, or thyroidectomy [1]. In our dataset of AUS cases, we can set the cut-off point of the diagnostic score at 0.523 to get the best overall diagnostic power (sensitivity: $87 \%$, specificity: $70.6 \%$ ) for the differentiation of benign and malignant tumors (Table 4). Less aggressive management such as repeated FNAC may be preferred for cases with diagnostic scores less than 0.523 . In contrast, more aggressive 
management such as thyroidectomy may be preferred for patients with diagnostic scores more than 0.523 . Or we can set the cut-off point at 0.209 to get the best sensitivity $(100 \%)$ for the purpose of avoiding unnecessary operation, which, in our dataset, avoids $13.6 \%$ (3 out of 22) of benign FN/SFN cases in our dataset from aggressive treatment. This scenario may also be used in the situation with the cytological diagnosis result of suspicions of malignancy (SUSP). We can set the diagnostic score of computerized cytomorphometry at 0.733 to get the best overall diagnostic power (sensitivity: $64 \%$, specificity: $100 \%$ ) (Table 4$)$. We could set the cut-off point at 0.426 to get the best sensitivity $(100 \%)$ while avoiding extensive thyroidectomy, i.e., 33\% (1 out of 3) of benign SUSP cases in our dataset, could have been exempted from aggressive treatment. In the situation of follicular neoplasm (FN)/suspicious of FN (SFN), the overall diagnostic power for differentiation of follicular adenoma (FA) and follicular thyroid carcinoma (FTC) is poor (Table 4). The comparison of the diagnostic score of FA and FTC was shown in Figure 2C. The ROC curve of the diagnostic score to differentiate benign and malignant lesions in FN/SFN was shown in Figure 1C with the AUC of 0.568 and $p$ value of 0.52 . However, if we set the cut-off point of the diagnostic score at 0.075 to get the best sensitivity $(100 \%)$, we could still avoid 13.6\% (3 out of 22) benign FN/SFN cases in our dataset from aggressive treatment.

The cytological features that can differentiate benign from malignant thyroid tumors in AUS/FLUS and SUSP categories of cytological specimens do not perform well for the category of FN/SFN. This indicates that the cytological characteristics of tumors with a follicular growth pattern are different from those of tumors with a papillary growth pattern. This is supported by the fact that the oncogenes associated with these two growth patterns are also different [18]. Since the diagnosis of follicular neoplasm depends on the histologic analysis of vascular and/or capsular invasion, the literature has reported that it is difficult to differentiate benign from malignant follicular neoplasms in cytological specimens [6] and it is not surprising to see that the computerized cytological features proposed by this study have also failed to differentiate the follicular neoplasms effectively.

In our study, the Red-Green-Blue (RGB) color space of image pixels were first converted into the color space of hue $(\mathrm{H})$, saturation $(\mathrm{S})$, and value $(\mathrm{V})$. To the best of our knowledge, this is the first study to evaluate the thyroid FNAC with indeterminate categories using the color space of HSV. In the study conducted by Celik ZE et al., morphological and chromatic variables were used to establish their computerized cytomorphometry system, which included five variables [19]. Among these five variables, only one was chromatic variable and was in grey scale. In another study [20], Gilshtein $\mathrm{H}$ et al. applied 50 variables in their computerized cytomorphometry, but the texture of nuclear chromatin was also in gray scale. In addition, our study collected a large dataset of 240 cases. Prior research included 125 cases of indeterminate cytological categories while the previous studies had only 40 and 58 cases, respectively.

The major limitation of this study is the small number of patients in each Bethesda category. In our dataset, more than $95 \%$ of PTC (105 out of 110 ) were CPTC and only five cases were follicular variants of PTC. We have noted that the CPTC constitutes $80 \%$ of all PTC in the literature [21] and further study is needed especially for the non-invasive follicular thyroid neoplasm with papillary like nuclear features (NIFTP) because of its different malignant potential [22]. For a more comprehensive clinical use, we should also collect a sufficient sample of other types of thyroid cancers, such as anaplastic thyroid cancer, poorly differentiated thyroid cancer, and medullary thyroid cancers, to be included in the analysis. However, the findings of the current study are still of value in differential diagnosis of PTC, which constitutes most thyroid cancers. Another concern is the use of Riu's stain, which is not widely used in other countries, and the proposed cytological features should be further evaluated using different stains. However, since the Riu's stain, which is also a type of Romanowsky stain [23], has the appearance resembling the Diff-Quik stain [24]. Similar results may be anticipated for the use of the Diff-Quik stain. However, different staining methods may result in different NCHR, NCSR, and NCVR values. We should collect and analyze specimens of other staining methods such as the Papanicolaou stain in the future. 


\section{Materials and Methods}

This study followed the ethical principles of the Declaration of Helsinki and was approved by the Institutional Review Board of the National Taiwan University Hospital (NTUH) (protocol number: 201603064RIPB). The study protocol with the waiver of informed consent has been approved by NTUH's research ethics committee and was registered on ClinicalTrials.gov (protocol number: NCT03105648).

\subsection{Study Subjects}

We recruited 244 cases who had pathologic diagnoses of benign nodules, PTC, follicular thyroid carcinoma (FTC), or Hurthle cell carcinoma, and had underwent FNAC by endocrinologists in NTUH between 2010 and 2017, which was followed by thyroidectomy. Three cases with a pathologic diagnosis of lymphocytic thyroiditis and 1 with a pathologic diagnosis of Graves' disease were excluded (Figure 3). The analysis of 240 cases included 110 cases with PTC, 100 with nodular/adenomatous goiter/hyperplasia, 10 with follicular/Hurthle cell carcinoma, and 20 with follicular adenoma.

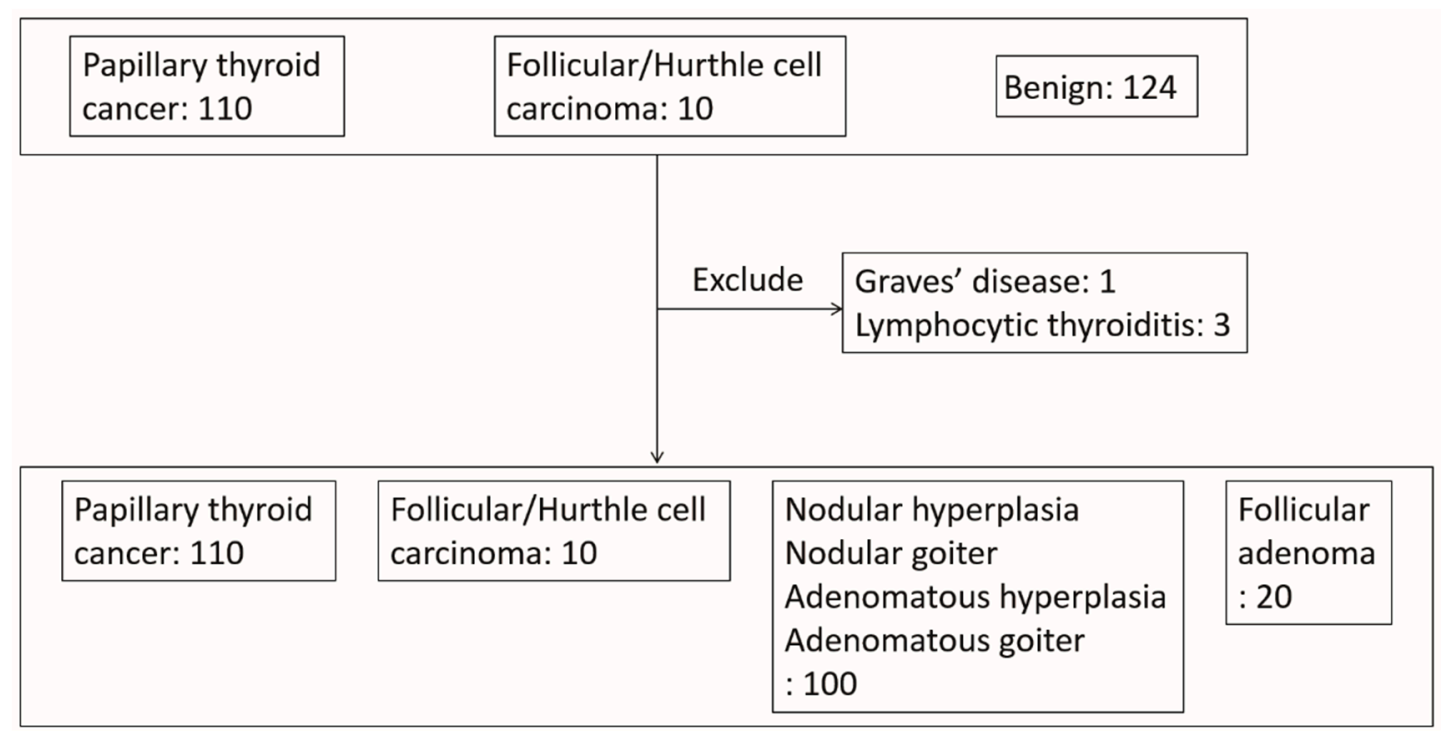

Figure 3. Flowchart of subject recruitment.

FNAC specimens underwent Riu's staining, which is a type of Romanowsky staining [23]. Riu's staining was performed in the following standard procedure. After air drying, the smears were covered with solution A $(0.05 \%$ methylene blue and $0.17 \%$ eosin $\mathrm{Y}$ in methanol) for $30 \mathrm{~s}$, which was followed by placement of solution $\mathrm{B}\left(0.12 \%\right.$ azure I, $0.14 \%$ methylene blue, $2.52 \% \mathrm{Na}_{2} \mathrm{HPO}_{4} \cdot 12 \mathrm{H}_{2} \mathrm{O}$, and $1.25 \%$ $\mathrm{KH}_{2} \mathrm{PO}_{4}$ in distilled water) for $90 \mathrm{~s}$. The stain mixtures were then washed off and the slides were observed under a light microscope. Digital images of these stained FNAC specimens were obtained and analyzed.

\subsection{Computerized Analysis of Cytologic Features}

Digital cytological images in matrices of color pixels were collected for computerized analysis (Figure 4). 

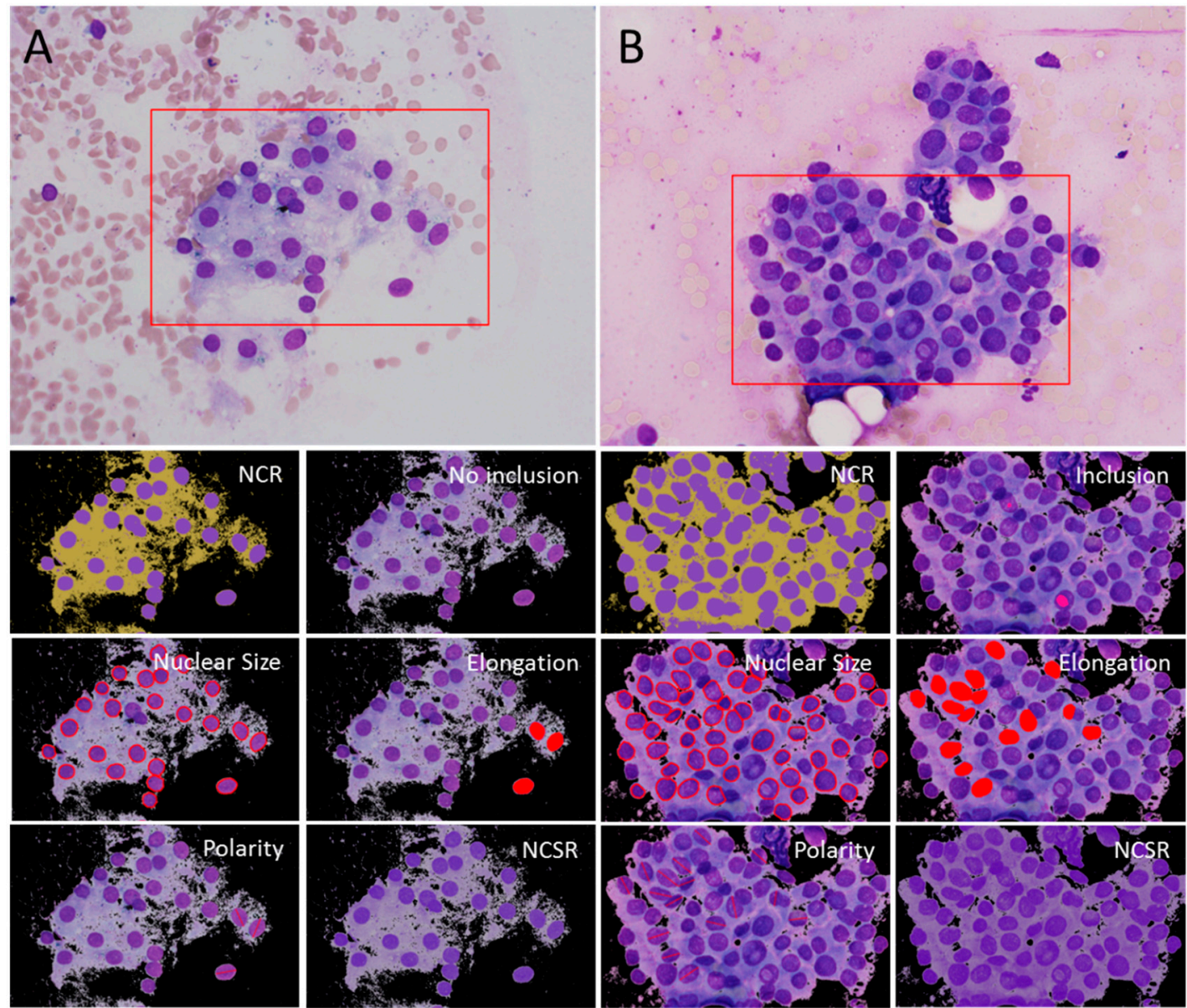

Figure 4. Visual comparison for clinically important features. The images from a case of hyperplasia (A) and a case of papillary thyroid carcinoma (B) acquired from a thyroid fine-needle aspiration cytology smear (Riu's stain, 400 $\times$ ). The image (red rectangle region of interest) was cropped for computerized analysis. NCR (nuclear-cytoplasmic ratio visualized with nuclei in purple, cytoplasm in yellow), intranuclear inclusion (pink), nuclear size (nuclei segmented by red contours), elongation (elongated nuclei in red), polarity (alignment of red major axes of elongated nuclei), and NCSR (nuclear-cytoplasmic saturation ratio visualized with a saturation contrast between nuclei and cytoplasm).

The image processing and analysis were performed using AmCAD-CA (AmCad BioMed Corp., Taipei, Taiwan). The digital cytological images were segmented into three clusters, including background, cytoplasm, and nucleus, based on the pixel value in hue and saturation with Otsu's method. After segmentation, the background cluster was set to zero, and the Canny edge detection was performed on the remaining image to find the boundary of nuclear area. The detailed algorithm used by the software can be seen in the patent [25] filed by the software company. The pixel values in the Red-Green-Blue (RGB) color space were first converted into the color space of hue (H), saturation $(\mathrm{S})$, and value (V). The $\mathrm{H}$ of nuclei and cytoplasm in the same cytologic specimen was compared and the difference was calculated in a nuclear-to-cytoplasmic hue ratio (NCHR) using a formula presented in the Supplementary Material. Nuclear-cytoplasmic saturation ratio (NCSR) and nuclear-cytoplasmic value ratio (NCVR) were generated in similar fashion. Nuclear-cytoplasmic ratio (NCR) was defined as the ratio of the area of the nuclei and cytoplasm. The segmented margin of the nuclei could then be used for statistical values, such as the sample mean (M), the sample standard deviation (SD), and the coefficient of variation $(\mathrm{CV}=\mathrm{SD} / \mathrm{M})$ of the morphological features including nuclear size, circularity, ellipticity, elongation, nuclear polarity, inclusion, and overlapping. Circularity indicated roundness of a cell nucleus by considering the nuclear perimeter and area. Ellipticity evaluated nuclear surface 
smoothness by considering axis lengths, perimeter, and area of the nucleus. Elongation assessed nuclear extension by considering both major and minor axis lengths of the nucleus. The overlapping index was defined as the ratio of overlapped nuclei to the total nuclear area. The index of nuclear polarity (NP) was defined as the variability of nuclear long-axis angles. The above calculating formulae were listed in the Supplementary Material.

\subsection{Statistical Analysis}

A total of 22 quantified cytological features, including 16 morphological and 6 chromatic features, were computed and analyzed. Analysis of variance and the Scheffé test were used to compare the differences in cytological features among 4 types of thyroid nodules based on a pathologic diagnosis. The D'Agostino-Pearson test was used to test for normal distribution of each feature. Multivariate logistic regression analysis was used to investigate the relationship between cytological features and pathologic results. Statistical analysis was performed using MedCalc Version 17.6 (MedCalc Software, Mariakerke, Belgium) and Stata/SE 14.0 for Windows (StataCorp LP, TX, USA). A $p$ value less than 0.05 was considered statistically significant.

\section{Conclusions}

Our study showed that quantification of cytological morphological and chromatic features could be used to develop a computer-aided tool for diagnosing thyroid nodules. We will recruit more cases and other types of thyroid cancer to improve the diagnostic scoring system and examine the cytological features of specimens prepared with other staining methods.

Supplementary Materials: The following are available online at http://www.mdpi.com/2072-6694/11/11/1645/s1, Computerized Analysis of Cytologic Features.

Author Contributions: Conceptualization, S.-R.S., A.C., I.-S.J., and T.-C.C. Methodology, A.C. and I.-S.J. Software, W.-Y.C. Validation, W.-Y.C. and A.C. Formal Analysis, W.-Y.C. and A.C. Investigation, S.-R.S. and A.C. Resources, S.-R.S. and I.-S.J. Data Curation, C.-Y.W., I.-S.J., T.-C.C., K.-Y.C., W.-Y.C., and Y.-L.H. Writing-Original Draft Preparation, S.-R.S., W.-Y.C., and A.C. Writing-Review \& Editing, S.-R.S., C.-Y.W., and A.C. Visualization, W.-Y.C. and A.C. Supervision, T.-C.C. Project Administration, S.-R.S. and A.C. Funding Acquisition, S.-R.S. and A.C.

Funding: The AmCad BioMed Corporation, Taipei, Taiwan, sponsored this study in terms of technical assistance and financial support.

Acknowledgments: We thank the staff of the Eighth Core Lab, Department of Medical Research, National Taiwan University Hospital, Liver Disease Prevention and Treatment Research Foundation, Taiwan, and Wong-Yuan Endocrine Fund, Taiwan, for their support during the study.

Conflicts of Interest: Y.-L.H. and C.-Y.W. have nothing to declare. S.-R.S., I.-S.J., K.-Y.C., and T.-C.C. received research grant support from AmCad BioMed. W.-Y.C. is employed by AmCad BioMed. A.C. was previously employed by and is now a consultant for AmCad BioMed.

\section{References}

1. Cooper, D.S.; Doherty, G.M.; Haugen, B.R.; Kloos, R.T.; Lee, S.L.; Mandel, S.J.; Mazzaferri, E.L.; McIver, B.; Pacini, F.; Schlumberger, M.; et al. Revised American Thyroid Association Management Guidelines for Patients with Thyroid Nodules and Differentiated Thyroid Cancer. Thyroid 2009, 19, 1167-1214. [CrossRef] [PubMed]

2. Bongiovanni, M.; Spitale, A.; Faquin, W.C.; Mazzucchelli, L.; Baloch, Z.W. The Bethesda System for Reporting Thyroid Cytopathology: A Meta-Analysis. Acta Cytol. 2012, 56, 333-339. [CrossRef] [PubMed]

3. Luu, M.H.; Fischer, A.H.; Pisharodi, L.; Owens, C.L. Improved preoperative definitive diagnosis of papillary thyroid carcinoma in FNAs prepared with both ThinPrep and conventional smears compared with FNAs prepared with ThinPrep alone. Cancer Cytopathol. 2011, 119, 68-73. [CrossRef] [PubMed]

4. Nayar, R.; Ivanovic, M. The indeterminate thyroid fine-needle aspiration: Experience from an academic center using terminology similar to that proposed in the 2007 National Cancer Institute Thyroid Fine Needle Aspiration State of the Science Conference. Cancer 2009, 117, 195-202. [CrossRef] [PubMed] 
5. Theoharis, C.G.; Schofield, K.M.; Hammers, L.; Udelsman, R.; Chhieng, D.C. The Bethesda Thyroid Fine-Needle Aspiration Classification System: Year 1 at an Academic Institution. Thyroid 2009, 19, 1215-1223. [CrossRef] [PubMed]

6. Haugen, B.R.; Alexander, E.K.; Bible, K.C.; Doherty, G.M.; Mandel, S.J.; Nikiforov, Y.E.; Pacini, F.; Randolph, G.W.; Sawka, A.M.; Schlumberger, M.; et al. 2015 American Thyroid Association Management Guidelines for Adult Patients with Thyroid Nodules and Differentiated Thyroid Cancer: The American Thyroid Association Guidelines Task Force on Thyroid Nodules and Differentiated Thyroid Cancer. Thyroid 2016, 26, 1-133. [CrossRef] [PubMed]

7. Shih, S.R.; Chang, Y.C.; Li, H.Y.; Liau, J.Y.; Lee, C.Y.; Chen, C.M.; Chang, T.C. Preoperative prediction of papillary thyroid carcinoma prognosis with the assistance of computerized morphometry of cytology samples obtained by fine-needle aspiration: Preliminary report. Head Neck 2013, 35, 28-34. [CrossRef]

8. Shih, S.-R.; Li, H.-Y.; Hsiao, Y.-L.; Chang, T.-C. Prognostic Significance of Cytologic Features in Fine-Needle Aspiration Cytology Samples of Papillary Thyroid Carcinoma: Preliminary Report. Thyroid 2006, 16, 775-780. [CrossRef]

9. Nikiforov, Y.E. Role of molecular markers in thyroid nodule management: Then and now. Endocr. Pract. 2017, 23, 979-988. [CrossRef]

10. Harrell, R.M.; Bimston, D.N. Surgical utility of Afirma: Effects of high cancer prevalence and oncocytic cell types in patients with indeterminate thyroid cytology. Endocr. Pract. 2014, 20, 364-369. [CrossRef]

11. Marti, J.L.; Avadhani, V.; Donatelli, L.A.; Niyogi, S.; Wang, B.; Wong, R.J.; Shaha, A.R.; Ghossein, R.A.; Lin, O.; Morris, L.G.T.; et al. Wide Inter-institutional Variation in Performance of a Molecular Classifier for Indeterminate Thyroid Nodules. Ann. Surg. Oncol. 2015, 22, 3996-4001. [CrossRef] [PubMed]

12. McIver, B.; Castro, M.R.; Morris, J.C.; Bernet, V.; Smallridge, R.; Henry, M.; Kosok, L.; Reddi, H. An Independent Study of a Gene Expression Classifier (Afirma) in the Evaluation of Cytologically Indeterminate Thyroid Nodules. J. Clin. Endocrinol. Metab. 2014, 99, 4069-4077. [CrossRef] [PubMed]

13. Wang, S.-L.; Wu, M.-T.; Yang, S.-F.; Chan, H.-M.; Chai, C.-Y. Computerized nuclear morphometry in thyroid follicular neoplasms. Pathol. Int. 2005, 55, 703-706. [CrossRef] [PubMed]

14. Schlumberger, M.-J.; Filetti, S.; Alexander, E.K.; Hay, I.D. Nontoxic diffuse goiter, nodular thyroid disorders, and thyroid malignancies. In Williams Textbook of Endocrinology; Elsevier: Amsterdam, The Netherlands, 2016; pp. 449-488.

15. Karbowniczek, M.; Chosia, M.; Domagała, W. Nuclear morphometry of MIB-1 positive and negative tumor cells in primary and metastatic malignant melanoma of the skin. Pol. J. Pathol. 1999, 50, 235-241.

16. Kazanowska, B.; Jelen, M.; Reich, A.; Tarnawski, W.; Chybicka, A. The role of nuclear morphometry in prediction of prognosis for rhabdomyosarcoma in children. Histopathology 2004, 45, 352-359. [CrossRef]

17. Paraskevakou, E.; Kavantzas, N.; Pavlopoulos, P.M.; Delibasis, A.; Yova, D.; Davaris, P. Computerized nuclear morphometry of renal cell carcinomas. Gen. Diagn. Pathol. 1996, 142, 101-104.

18. Nikiforov, Y.E.; Seethala, R.R.; Tallini, G.; Baloch, Z.W.; Basolo, F.; Thompson, L.D.R.; Barletta, J.A.; Wenig, B.M.; Al Ghuzlan, A.; Kakudo, K.; et al. Nomenclature Revision for Encapsulated Follicular Variant of Papillary Thyroid Carcinoma: A Paradigm Shift to Reduce Overtreatment of Indolent Tumors. JAMA Oncol. 2016, 2, 1023-1029. [CrossRef]

19. Celik, Z.E.; Altinay, S.; Kilinc, F.; Arslan, N.; Yilmaz, B.S.; Karabagli, P.; Ugurluoglu, C. Using Computerized Cytomorphometry to Distinguish between Benign and Malignant Cases in Thyroid Fine-Needle Aspiration Cytology. Diagn. Cytopathol. 2016, 44, 902-911. [CrossRef]

20. Gilshtein, H.; Mekel, M.; Malkin, L.; Ben-Izhak, O.; Sabo, E. Computerized cytometry and wavelet analysis of follicular lesions for detecting malignancy: A pilot study in thyroid cytology. Surgery 2017, 161, 212-219. [CrossRef]

21. Gupta, S.; Ajise, O.; Dultz, L.; Wang, B.; Nonaka, D.; Ogilvie, J.; Heller, K.S.; Patel, K.N. Follicular variant of papillary thyroid cancer: Encapsulated, nonencapsulated, and diffuse: Distinct biologic and clinical entities. Arch. Otolaryngol. Head Neck Surg. 2012, 138, 227-233. [CrossRef]

22. Bongiovanni, M.; Faquin, W.; Giovanella, L.; Durante, C.; Kopp, P.; Trimboli, P. Impact of non-invasive follicular thyroid neoplasms with papillary-like nuclear features (NIFTP) on risk of malignancy in patients undergoing lobectomy/thyroidectomy for suspicious for malignancy or malignant fine-needle aspiration cytology findings: A systematic review and meta-analysis. Eur. J. Endocrinol. 2019. [CrossRef] 
23. Riu, C.H. A new rapid method of staining thin blood film: First report. Taiwan I Hsueh Hui Tsa Chih. 1953, 52, 348-352.

24. Wright, J.H. A Rapid Method for the Differential Staining of Blood Films and Malarial Parasites. J. Med. Res. 1902, 7, 138-144. [PubMed]

25. Chen, A.; Hsiao, Y.H.; Chang, T.C.; Jan, I.S.; Shih, S.R.; Wang, H.M. Cytological Image Processing Device, and Method for Quantifying Characteristics of Cytological Image. Patent Number TWI630581B, 21 July 2018.

(C) 2019 by the authors. Licensee MDPI, Basel, Switzerland. This article is an open access article distributed under the terms and conditions of the Creative Commons Attribution (CC BY) license (http://creativecommons.org/licenses/by/4.0/). 